\title{
IDENTIFICACIÓN MACRO Y MICROSCÓPICA DE GRANOS DE ZEA MAYS (POACEAE) EN CONTEXTOS PREHISPÁNICOS TEM PRANOS DE LA QUEBRADA DE LOS CORRALES (TUCUMÁN, ARGENTINA)
}

\author{
Nurit Oliszewski', Rocío Molar², Guillermo Arreguez¹, Julieta Carrizo³ \& Jorge G. Martínez
}

${ }^{1}$ Instituto Superior de Estudios Sociales, ISES-CONICET, Universidad Nacional de Tucumán, San Lorenzo 429, 4000, San Miguel de Tucumán, Tucumán, Argentina; nuritoli@yahoo.com.ar (autor corresponsal).

${ }^{2}$ Instituto de Estudios Históricos, IEH-CONICET, Universidad Nacional de Córdoba, Córdoba, Argentina.

${ }^{3}$ Facultad de Ciencias Naturales e IML, Universidad Nacional de Tucumán, Tucumán, Argentina.

\begin{abstract}
Oliszewski, N.; R. Molar, G. Arreguez, J. Carrizo \& J. G. Martínez. 2019. Macro and microscopic identification of Zea mays grains (Poaceae) in early prehispanic contexts of Quebrada de Los Corrales (Tucumán, Argentina). Darwiniana, nueva serie 7(1): 5-15.

In this work we report the discovery of charred macrorrests of Zea mays grains from early contexts (ca. 3500 years BP) of the archaeological site Taller Puesto Viejo 1, in Quebrada de Los Corrales (Tucumán, Northwest Argentina). For the identification, a combined analysis of both macroscopic diagnostic characters (texture, structure and fracturing pattern) and microscopic characters (starch granules and phytoliths) was implemented. This methodology allowed the identification of nine carbonized corn fragments that, at the microscopic level, have numerous starch granules and some phytoliths, diagnostic of the taxon. We evaluate the implications of this finding in its context of mortuary type and in the general context of evidence of early maize in Argentina and Chile.
\end{abstract}

Keywords. Archaeobotanical macrorremains; archaeobotanical microrremains; prehispanic corn; Quebrada de Los Corrales; Tucumán.

Resumen. Oliszewski, N.; R. Molar, G. Arreguez, J. Carrizo \& J. G. Martínez. 2019. Identificación macro y microscópica de granos de Zea mays (Poaceae) en contextos prehispánicos tempranos de la Quebrada de Los Corrales (Tucumán, Argentina). Darwiniana, nueva serie 7(1): 5-15.

En el presente trabajo damos a conocer el hallazgo de macrorrestos carbonizados de granos de Zea mays procedentes de contextos tempranos (ca. 3500 años AP) del sitio arqueológico Taller Puesto Viejo 1, en la Quebrada de Los Corrales (Tucumán, Argentina). Para la identificación se implementó un análisis combinado de caracteres diagnósticos tanto macroscópicos (textura, estructura y patrón de fracturación) como microscópicos (gránulos de almidón y fitolitos). Esta metodología permitió identificar nueve fragmentos de maíz carbonizados que, a nivel microscópico, poseen numerosos gránulos de almidón y algunos fitolitos diagnósticos del taxón. Se evalúan las implicancias de este hallazgo en el contexto mortuorio en que fueron recuperados y en el contexto general de evidencias del maíz temprano de Argentina y Chile.

Palabras clave. Macrorrestos arqueobotánicos; maíz prehispánico; microrrestos arqueobotánicos; Quebrada de Los Corrales; Tucumán. 


\section{INTRODUCCIÓN}

La presencia de maíz es recurrente en los sitios arqueológicos prehispánicos del noroeste de Argentina (NOA) durante el Holoceno Tardío, siendo uno de los granos alimenticios registrado con mayor frecuencia. Ha sido hallado tanto en forma de desechos de consumo asociado a pisos de ocupación y fogones, así como microrrestos en contextos rituales y en artefactos de molienda, es decir en contextos de procesamiento. A pesar de ser un cultivo que se desarrolla mejor en tierras de baja y de mediana altitud (hasta los $2000 \mathrm{~m} \mathrm{~s}$. $\mathrm{m}$.), ha sido identificado en sitios arqueológicos ubicados a 3000 e incluso a más de $3500 \mathrm{~m} \mathrm{~s}$. m. como en Antofagasta de la Sierra donde se ha podido determinar la presencia de distintas variedades con cronologías de entre ca. 1970 y 530 años AP (Rodríguez \& Aschero 2007). Su frecuencia aumenta notablemente durante el primer milenio de la Era Cristiana asociado a sitios correspondientes a la etapa agro-pastoril plena en contextos aldeanos, con todo lo que ello implica en cuanto a su producción. En consonancia, en esta etapa se multiplican los hallazgos tanto de micro como de macrorrestos en diversos contextos. Es importante destacar que alrededor de 1500 años AP se habría producido la incorporación de nuevas razas de maíz en el NOA (Oliszewski, 2012). Durante el segundo milenio, el maíz acompañó el proceso de intensificación agrícola siendo numerosos los sitios que cuentan con evidencias de su presencia. Para una puesta al día sobre el maíz como recurso prehispánico en el norte de Argentina y de Chile se puede consultar, entre otros: Rodríguez \& Aschero (2007); Korstanje \& Cuenya (2010); Oliszewski (2012); Franco Salvi et al. (2014); Lema (2014); McRostie (2014); Ratto et al. (2014); Aguirre \& Rodríguez (2015).

Los datos no dejan dudas acerca de la importancia que tuvo este cultivo en el devenir de las sociedades prehispánicas tanto del NOA como del norte de Chile. El interrogante es cuándo y cómo estos grupos incorporaron el maíz a su modo de vivir, en particular dentro del NOA. Cabe aclarar que como toda la evidencia corresponde inequívocamente a $\mathrm{Zea}$ mays L., y no hay teosintes nativos, esta región queda al margen de las discusiones acerca de los procesos de domesticación de esta especie. Sin embargo, es interesante evaluar cuándo comenzó a ser manipulado y con qué objetivos: ¿alimenticio?, ¿ritual?, ¿ambos?

En este marco, los objetivos de este trabajo son, en primer lugar, dar a conocer el hallazgo de macrorrestos carbonizados de Zea mays en el sitio Taller Puesto Viejo 1 (TPV1) en la Quebrada de Los Corrales (Tucumán, Argentina) con una cronología temprana de ca. 3500 años AP, y, por otra parte, evaluar las implicancias de este hallazgo en el contexto general de evidencias del maíz temprano de Argentina y Chile.

\section{Los primeros registros de Zea mays en el norte de Argentina y Chile}

Para el área andina, tal como ocurre en los sitios Paredones y Huaca Prieta en Perú, se han registrado fitolitos y gránulos de almidón para el intervalo 6775-6500 años AP y macrorrestos para el lapso 5000-3600 años AP (Grobman et al., 2012). Para el norte de Argentina y Chile los primeros registros de maíz son dos mil años más tardíos y corresponden a fitolitos y gránulos de almidón provenientes de artefactos de molienda del sitio Quebrada Seca 3 situado en la Puna de Catamarca (Argentina), asociados a fechados de entre 5000 y 4600 años AP (Babot, 2011).

En el norte de Chile, en la Quebrada de Tulán se reportaron molinos en el sitio Tulán 54 -ca. 3400 y 2400 años AP- que contienen gránulos de almidón de Z. mays, mientras que las evidencias más antiguas de macrorrestos proceden de Tulán 85 con una cronología de 2660 años AP (Núñez et al., 2009).

Para el NOA las evidencias más tempranas de macrorrestos fueron datadas entre 2100 y 1900 años AP y provienen de un montículo artificial de probable función ceremonial sito en El Mollar, Tucumán (González y Núñez Regueiro 1960; Carrizo et al., 1999) a sólo 22 $\mathrm{km}$ al sur de TPV1. Desde los inicios de la Era Cristiana, como ya se mencionó, las evidencias macroscópicas se multiplican. En resumen, los escasos registros tempranos para el norte de Argentina y de Chile indican presencia de maíz desde ca. 5000-4600 años AP (microrrestos) y desde ca. 2660 años AP (macrorrestos). 
Marco general de la Quebrada de Los Corrales y el registro de maíz

Las investigaciones en la Quebrada de Los Corrales (QDLC), que se iniciaron en 2005, permitieron identificar diversos tipos de evidencias arqueológicas correspondientes a distintos momentos de ocupaciones humanas que se iniciaron en el Holoceno Medio (ca. 7800 años AP) y concluyeron a mediados del segundo milenio de la Era Cristiana (ca. 650 años AP) (Martínez et al., 2013, 2016; Oliszewski et al., 2015). De particular interés es el período 40002000 años AP correspondiente a la transición entre grupos cazadores y grupos productores de alimentos. Hemos propuesto la ocurrencia en QDLC de un proceso transicional local que llevó paulatinamente a grupos cazadores-recolectores móviles (ca. 3800-3500 años AP) a constituirse como una aldea agro-pastoril conformada por casi un centenar de viviendas de diseño "patrón Tafí" que tuvo su auge de ocupación entre 1850 y 1550 años AP (Oliszewski et al., 2018). Hacia 1500 años AP, las ocupaciones humanas en esta zona se interrumpieron abruptamente por la probable ocurrencia de un evento volcánico que habría inutilizado la producción agrícola y el agua para consumo humano y animal. A partir de ese momento, QDLC habría funcionado sólo como un espacio de circulación sin ocupación/producción efectiva y/o como una eventual área estacional de pastoreo de llamas (Oliszewski et al., 2015).

QDLC es una microrregión arqueológica situada por encima de los $3000 \mathrm{~m} \mathrm{~s}$. m. en el sector norte de las Sierras del Aconquija, más precisamente en el abra de El Infiernillo (oeste de la provincia de Tucumán). El área de estudio tiene una superficie de $28 \mathrm{~km}^{2}$ y comprende a la cuenca inferior, media y superior del río de Los Corrales (Figura 1). En la cuenca inferior se ubican Cueva de Los Corrales 1 y 2, en la cuenca media/superior se registran extensas áreas con estructuras agrícolas y pastoriles y finalmente en ambas márgenes del curso superior, en la localidad arqueológica de Puesto Viejo (PV1 y $\mathrm{PV} 2$ ), se concentran 85 unidades residenciales conformando un gran núcleo aldeano. Hacia el sur de PV se encuentra el mencionado sitio Taller Puesto Viejo 1 que tiene la particularidad de contar con una larga persistencia ocupacional (ca. 7800 - 1750 años AP). Las evidencias arqueobotánicas bajo análisis proceden de este sitio el cual se ubica en la margen oeste del río Los Corrales y consiste en una concentración de evidencias prehispánicas en un espacio a cielo abierto $(34 \times 24 \mathrm{~m})$ delimitado por unidades residenciales del $1^{\text {er }}$ milenio de la Era Cristiana (Figura 1). Su principal característica es la singularidad de presentar materiales arqueológicos en estratigrafía asignables al Holoceno Medio, hecho poco frecuente en el área de valles y quebradas del NOA para dichas cronologías (Oliszewski et al., 2018). TPV1 ha sido definido como una base residencial donde, hacia 3800-3500 años AP, se llevaron a cabo, además de actividades domésticas de confección, mantenimiento y uso de artefactos varios de cerámica y piedra, prácticas funerarias consistentes en cremaciones realizadas en estructuras de combustión y acompañadas de elementos diversos como cuentas de collar líticas con decoraciones incisas, de moluscos marinos y alimentos de origen vegetal. Entre los últimos se determinó la presencia de semillas de quínoa (Chenopodium quinoa Willd.) que a pesar de encontrarse carbonizadas, preservaron muy bien sus rasgos diagnósticos específicos (Martínez et al., 2017; Oliszewski et al., 2018). Pero no ocurrió lo mismo con otros especímenes hallados en este mismo contexto, los cuales motivaron el presente trabajo: entre la matriz arenosa y mezclados con otros restos orgánicos e inorgánicos de tamaño pequeño, destacaban algunos fragmentos vegetales carbonizados que, por su textura y estructura, parecían corresponder a fragmentos de granos de maíz que se diferenciaban claramente de otros elementos carbonizados (materiales leñosos). Por este motivo se decidió realizar un abordaje múltiple que incluyó análisis macro y microscópicos así como también una datación directa de ${ }^{14} \mathrm{C}$ por AMS de uno de los fragmentos.

Además de estos hallazgos tempranos, destacamos que el maíz acompañó el devenir de las ocupaciones en QDLC durante la etapa agro-pastoril desde 1850 y hasta 650 años $\mathrm{AP}$, ya sea en forma de macrorrestos o de microrrestos y en distintos tipos de locaciones: estructuras residenciales, cueva y estructuras productivas, dando indicios de su cultivo in situ por encima de los $3000 \mathrm{~m} \mathrm{s.} \mathrm{m.}$ 


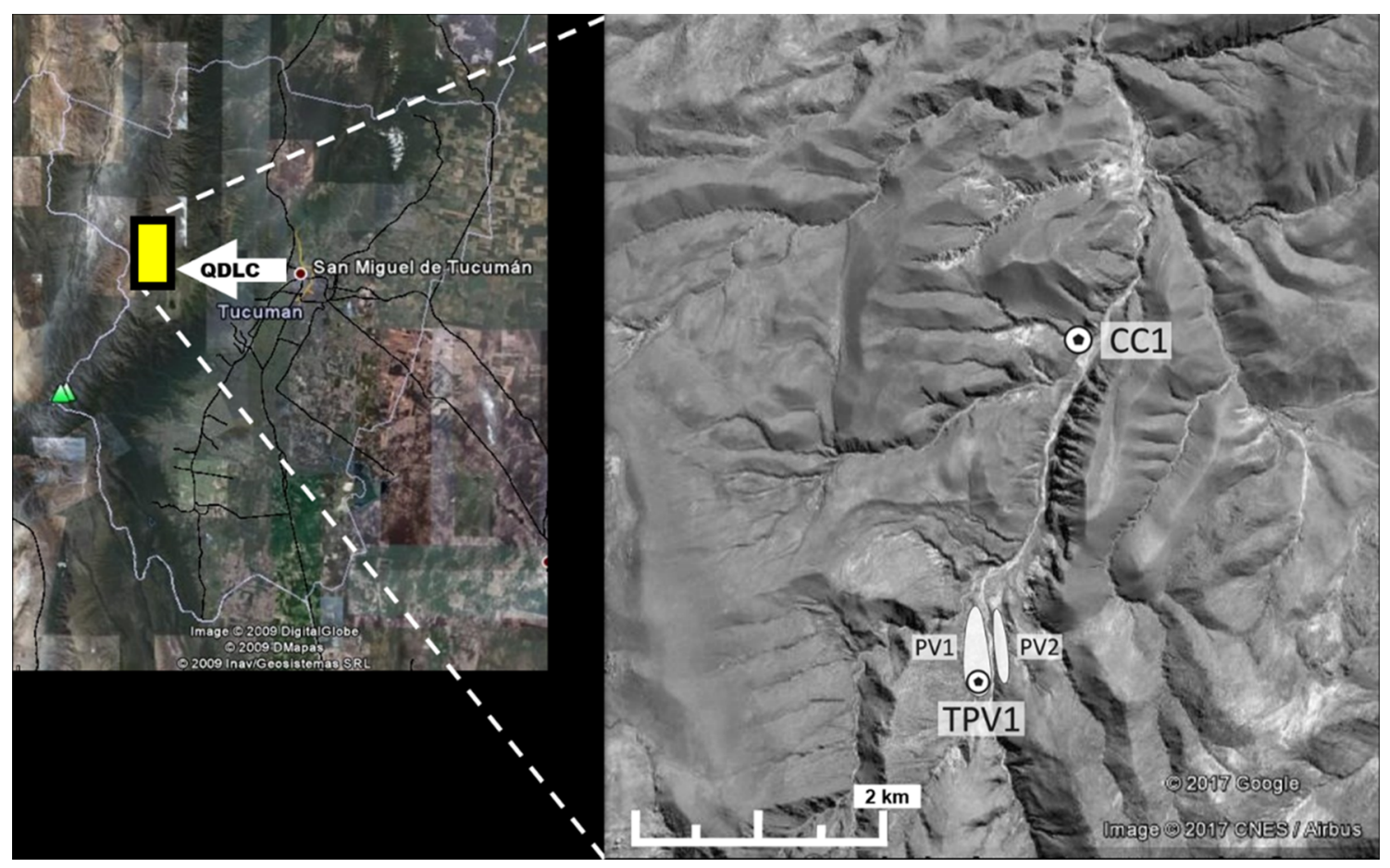

Fig. 1. Ubicación geográfica del área de procedencia de los especímenes de Z. mays de TPV1 (Quebrada de Los Corrales, El Infiernillo, Tucumán). PV1 y PV2: Puesto Viejo; CC1: Cueva de Los Corrales 1. Figura en color en la versión en línea http://www.ojs.darwin.edu.ar/index.php/darwiniana/article/view/806/1160

En dos de las estructuras residenciales del núcleo aldeano de Puesto Viejo ${ }^{1}$ (ca. 1770-1670 años AP) y formando parte de eventos de cocina, se encontraron granos y fragmentos de maíz carbonizados mezclados con semillas/endocarpos de otras plantas (quínoa, chañar y algarrobo) además de otras evidencias que confirman el carácter doméstico: núcleos, lascas y escasos artefactos líticos formatizados, artefactos de molienda, material cerámico y restos faunísticos correspondientes a camélidos (llama y guanaco), cérvidos (taruca) y dasipódidos (quirquincho) (Oliszewski y Arreguez, 2015). Además, los análisis isotópicos realizados sobre restos óseos humanos datados en ca. 3300, 2100 y 1560 años AP muestran una tendencia paulatina al aumento del consumo de maíz (Oliszewski y Martínez, 2017).

La cueva mencionada, localizada en el curso inferior del río, refiere al sitio Cueva de Los Corrales (CC1) que presenta la particularidad de poseer una secuencia estratigráfica primaria y catorce morteros confeccionados en la roca de base. Esta cueva habría sido utilizada para la realización de múltiples actividades -procesamiento, consumo y desecho de vegetales alimenticios entre ellas- pero no fue utilizada estrictamente como un espacio doméstico. En cuanto a su ubicación temporal CC1 tuvo dos momentos de ocupación: las primeras ocupaciones habrían ocurrido hacia ca. 2100 años AP y las últimas hacia $c a$. 650-630 años AP (Oliszewski et al., 2008). Para ambos momentos, y procedentes tanto de estratigrafía como de los artefactos de molienda, se registraron restos de chala, marlos y granos de maíz secos. Para momentos tempranos se identificó una única raza: Pisingallo (maíz de tipo duro), mientras que para los momentos tardíos se suman maíces de tipo semiduro y harinoso (Oliszewski, 2012). El maíz aparece para ambos momentos acompañado por semillas de quínoa ${ }^{2}$ y endocarpos de algarrobo y chañar; para las primeras ocupaciones se ha registrado también zapallo y para las más tardías poroto común (Arreguez et al., 2010). Además, en 
un molino móvil registrado en superficie, Babot (2007) identificó gránulos de almidón de maíz, pseudocereales y tubérculos microtérmicos.

Por último las estructuras productivas de QDLC están conformadas por un extenso sistema de andenería a secano y numerosos corrales que se ubican separados de las viviendas cubriendo una superficie de 500 hectáreas aproximadamente. Estas estructuras fueron contemporáneas a las viviendas del primer milenio de la Era Cristiana. La sincronía en el uso ha sido corroborada por una serie de evidencias recuperadas en excavaciones realizadas en un sector dentro de las áreas productivas. Una datación sobre hueso de camélido proveniente de dichas excavaciones, proporcionó un resultado de $1840 \pm 25$ años AP que es contemporáneo y coincidente con las primeras ocupaciones registradas para la aldea de Puesto Viejo (Oliszewski, 2017).

Con respecto a los taxones que podrían haberse cultivado, en sedimentos procedentes de andenes se detectó la presencia de fitolitos de la familia Poaceae que tienen afinidad con los que se citan para Z. mays (Gómez Augier et al., 2008).

Esta breve síntesis muestra que la presencia del maíz fue persistente en la historia ocupacional de QDLC.

\section{MATERIALES Y MÉTODOS}

Los materiales bajo estudio provienen de un contexto particular: estructuras de combustión o pozones funerarios, los cuales contenían en su interior una gran cantidad de restos óseos humanos quemados y altamente fragmentados mezclados con distintos tipos de restos orgánicos e inorgánicos en una matriz areno-carbonosa (Oliszewski et al., 2018). La particularidad de estas estructuras arqueológicas y de su contenido llevó a poner en práctica una metodología de recuperación durante la excavación en la cual todo el sedimento con sus materiales fue embolsado de manera completa para su posterior separación en laboratorio. Esta modalidad, que implica una meticulosa observación, fue aplicada con éxito antes en Cueva de Los Corrales 1, detectándose gracias a este procedimiento pequeñas semillas incluso menores a $1 \mathrm{~mm}$, las cuales se encontraban mezcladas con material lítico, restos óseos, partes de insectos, vellones y plumas en un sedimento arenoso (Arreguez et al., 2014). A partir de esta experiencia se decidió separar de forma manual en laboratorio el material de los pozones de TPV1 ya que, durante las tareas de excavación no podrían haber sido detectados y recuperados los macrorrestos arqueobotánicos de tamaño pequeño. De este modo, se recuperaron semillas de quínoa y cactáceas (Oliszewski et al., 2018) así como también pequeños fragmentos carbonizados que por su apariencia externa parecían ser fragmentos de granos de maíz.

Estos especímenes fueron analizados y fotografiados con lupa estereoscópica marca Zeiss con cámara digital (Axiocam Erc $5 \mathrm{~s}$ ) a diferentes aumentos. Los materiales arqueológicos fueron comparados con material de colecciones de referencia actual y arqueológico (en este último caso especialmente granos de maíz termoalterados provenientes de QDLC) y bibliografía específica para la identificación de granos de maíz (Parodi, 1959; Torregrosa et al., 1980; Oliszewski, 2009, 2012).

Se seleccionó el espécimen de mayor tamaño $(5 \times 3,5 \mathrm{~mm})$. Luego de retirarse una parte para datación, del mismo espécimen se extrajo con bisturí y pinza un pequeño fragmento para ser observado mediante el uso de microscopio. Dicho fragmento fue molido en un pequeño mortero (objeto que previamente fue sometido a remojo durante 1 hora y enjuagado con agua destilada, para evitar contaminaciones) y lo obtenido fue montado en un portaobjetos con aceite de inmersión. Se decidió proceder de esta manera para que los microcarbones se disgreguen y permitan corroborar la presencia/ ausencia de fitolitos y almidones. El análisis fue realizado con microscopio óptico trinocular con polarizador marca Biotraza, modelo XP-148PLT y las fotografías tomadas con cámara incorporada marca Arcano de 5.0 megapíxeles. Para esto se utilizó el programa IS-CAPTURE, software que permite procesar las imágenes a medida que se las va tomando, pudiendo realizar de este modo ajustes de luz, de medición de objetos, de saturación y de balance de blancos para destacar determinados elementos. 


\section{RESULTADOS}

La muestra se compone de nueve especímenes carbonizados que se encuentran depositados en el Instituto Superior de Estudios Sociales (CONICET). Los tamaños varían entre $5 \times 3,5$ y $2 \times 2 \mathrm{~mm}$, predominando los de $3 \times 2 \mathrm{~mm}$ y $2 \times 2 \mathrm{~mm}$ (Figura 2A y B). Los especímenes recuperados proceden de las Cuadrículas AII (Niveles 13 y $14 ; 85-95 \mathrm{~cm}$ de profundidad) y CI (Nivel 15 ; 95 $100 \mathrm{~cm}$ de profundidad). El estado fragmentario impidió la preservación de los caracteres diagnósticos primarios tales como dimensiones, forma y dureza que han permitido en otros casos arqueológicos la asignación a $Z$. mays e incluso la identificación de razas.

Los caracteres macroscópicos que llevaron a asignar a priori a estos fragmentos como Zea mays L. son la textura brillosa debido al alto contenido en azúcares que caracteriza a los granos de esta planta, la estructura interna que se presenta porosa y con oquedades y el patrón de fracturación curvo (Figura 3). Estos caracteres diagnósticos, que podemos denominar secundarios, permitieron determinar que se trataba de fragmentos de la parte interna de granos de maíz.
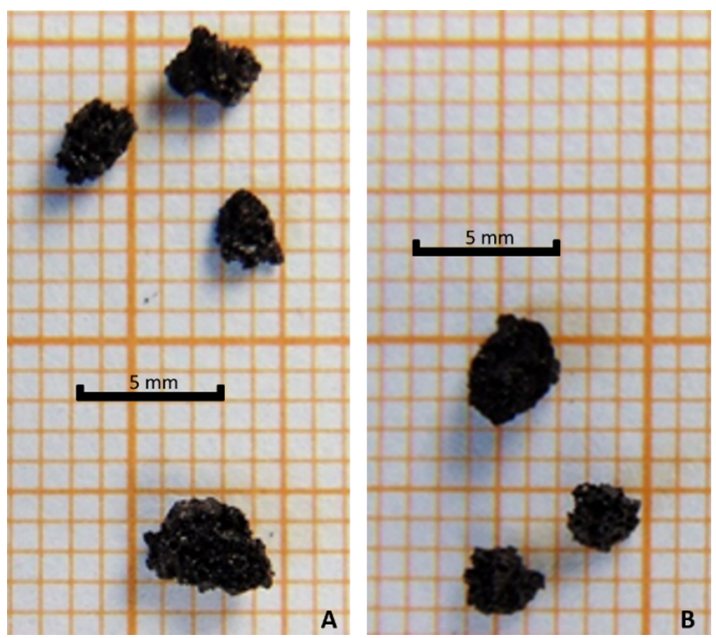

Fig. 2. Macrorrestos fragmentados de granos de Z. mays de TPV1 (Sector I, Cuadrícula+ AII). A) Nivel 13; B) nivel 14. Figura en color en la versión en línea http:// www.ojs.darwin.edu.ar/index.php/darwiniana/article/ view/806/1160

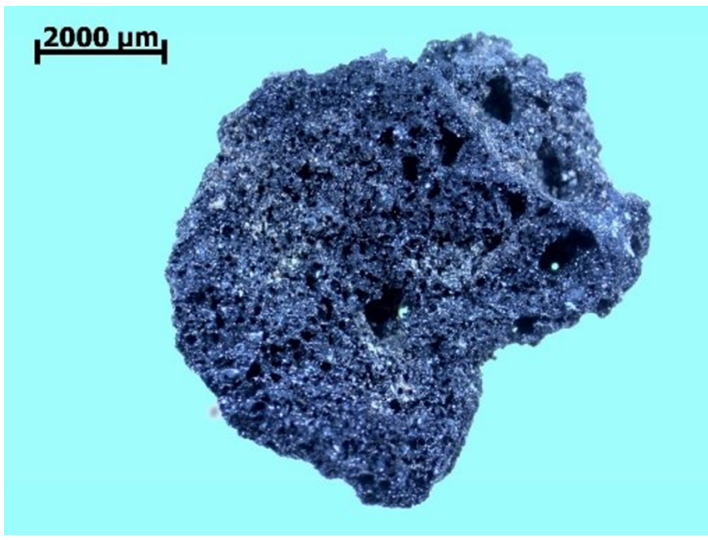

Fig. 3. Macrorresto fragmentado de grano de Z. mays de TPV1 (Sector I, Cuadrícula AII Nivel 13). Se observan los caracteres secundarios que permitieron la identificación: textura brillosa, estructura interna porosa y patrón de fragmentación curvo. Figura en color en la versión en línea http://www.ojs.darwin.edu.ar/index.php/darwiniana/article/view/806/1160

En trabajos anteriores realizados sobre granos de maíz de sitios arqueológicos de Catamarca (Campo del Pucará) y La Rioja (Loma Pircada) la textura y la estructura interna demostraron ser variables complementarias muy útiles para la identificación de granos carbonizados (Oliszewski, 2005; Carrizo et al., 2002). Para complementar la identificación taxonómica, debido especialmente a la cronología tan temprana del contexto de hallazgo - ca. 38003500 años AP-, se procedió a realizar un análisis microscópico del fragmento de mayor tamaño $(5 \times 3,5 \mathrm{~mm})$ procedente de la Cuadrícula AII, Nivel 13. Previamente se separó una parte del mismo para ser datado por ${ }^{14} \mathrm{C}$ (AMS), análisis que arrojó un fechado de $3520 \pm 25$ años AP (UGA\# 32586) $(3697-3826 \mathrm{cal}$ AP; valor medio $=3756 \mathrm{AP})$.

El análisis microscópico de esta muestra dio como resultado la presencia de un total de 38 gránulos de almidón, los cuales se clasificaron teniendo en cuenta su tamaño, morfología, cruz de extinción y atributos del hilum (Babot, 2003, 2006; Korstanje \& Babot, 2007; Lantos et al., 2014). De los 38 gránulos, 32 pudieron ser identificados como Zea mays L. (Figuras 4A y B). De forma esférica (94\%) o facetada (6\%), sus medidas varían entre 4 y $35 \mu \mathrm{m}$ (el $85 \%$ con medidas menores a 7,5 $\mu \mathrm{m}$ ), con hilum en forma de punto o similar a una pequeña hendidura. 


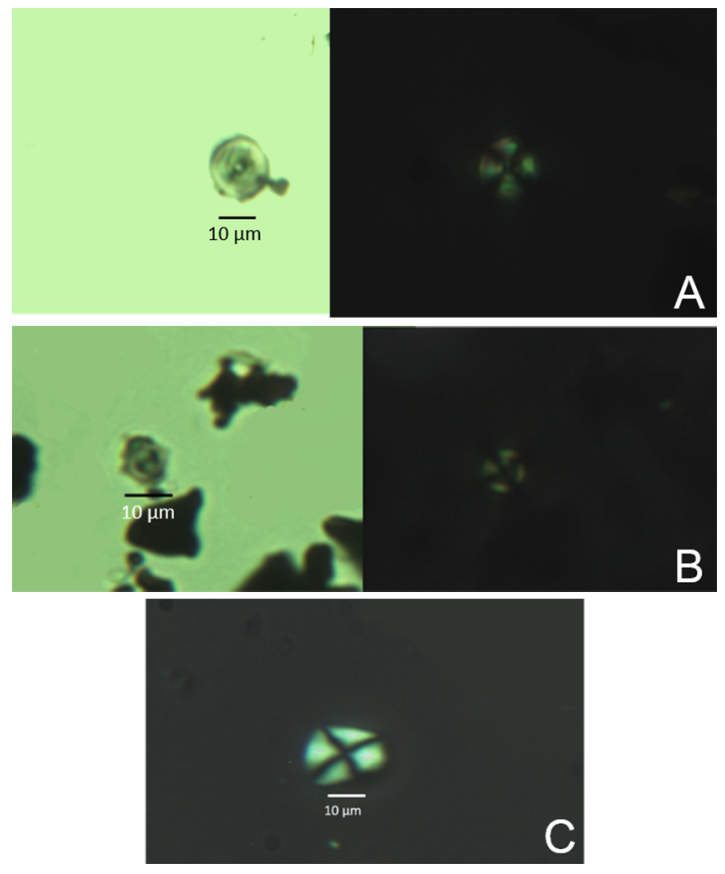

Fig. 4. Microfósiles de gránulos de almidón de Z. mays de TPV1 (Sector I, Cuadrícula AII Nivel 13) observados a 60X: A) y B) gránulos de almidón observables con campo claro (a la izquierda) y luz polarizada (a la derecha); C) gránulo de almidón observable únicamente con luz polarizada. Figura en color en la versión en línea http://www.ojs.darwin.edu.ar/index.php/darwiniana/ article/view/806/1160

En los 32 gránulos se pudo observar claramente la cruz de extinción con ángulos de $90^{\circ}$, presentándose los cuatro brazos con un brillo intenso y con punto de partida en el hilum. Los seis gránulos restantes podrían pertenecer a $Z$. mays, pero inconvenientes del muestreo y observación no permitieron afirmarlo rotundamente. Con estos inconvenientes nos referimos fundamentalmente a la alta presencia de microcarbones que dificultó en algunos casos descubrir o destapar los almidones que asomaban detrás, siendo observable sólo una parte del grano. Uno de estos granos presentaba las características propias de los granos de almidón de Z. mays, pero la cruz se observaba difuminada, muy difícil de percibir con nitidez. En otro caso, solo pudo observarse la cruz con luz polarizada, sin poder diferenciarse los contornos del grano en el campo claro (Figura 4C). Por otra parte, pudieron visualizarse e identificarse dos fitolitos, dificultando esta tarea nuevamente la cantidad de microcarbones presentes. Ambos se encontraban con algunas sustancias/elementos adheridos, sin embargo, es posible afirmar que son wavy top rondel, lo que permite asignarlos a Zea mays L. (Pearsall, 1989; Piperno, 2006). Además del fragmento de mayor tamaño que fuera fechado, en el resto de los especímenes también se identificaron gránulos de almidón.

En síntesis, el análisis conjunto de caracteres macro y microscópicos realizado permitió identificar a los nueve especímenes bajo estudio como fragmentos de la parte interna de granos de Zea mays L. El estado de carbonización y la alta fragmentación dificultaba la identificación exclusivamente a partir del análisis macroscópico. Ante la ausencia de los caracteres diagnósticos tradicionales para granos de maíz termoalterados (dimensiones y forma) a los cuales hemos denominado primarios, los caracteres secundarios resultaron ser sumamente útiles para la identificación taxonómica. Dichos caracteres son la textura, la estructura interna de los granos y el patrón de fragmentación (variable que introducimos en el presente trabajo). El modo de fragmentación distingue a los granos de maíz que presentan sus bordes curvados de otros macrorrestos carbonizados como las semillas de algarrobo o los frutos de chañar que presentan sus bordes rectos.

Sin embargo, y aún con estas variables macroscópicas, fue determinante para la identificación el análisis microscópico, ya que pudo constatarse la presencia fehaciente de gránulos de almidón (mayoritariamente) y fitolitos asignables a Z. mays. Respecto al tamaño, es de destacar que los gránulos son pequeños en comparación con otros identificados en materialidades como recipientes cerámicos provenientes del mismo sitio ya que en líneas generales, los gránulos de almidón de maíz procedentes del raspado de restos cerámicos, tienen tamaños de entre 7 y $21 \mu \mathrm{m}$, mientras que los gránulos bajo estudio son mayoritariamente menores a 7,5 $\mu \mathrm{m}$. Pero cabe destacar que, estudios realizados por otros autores afirman que esta reducción del diámetro puede deberse al sometimiento a altas temperaturas (Babot, 2003; Arriaza et al., 2015) lo cual es coherente con un contexto de cremación como el aquí tratado que se discutirá a continuación. 


\section{DISCUSIÓN Y CONCLUSIONES}

Los especímenes vegetales que analizamos corresponden a fragmentos de granos de maíz datados en ca. 3520 años AP, procedentes de un contexto funerario de cremación detectado en el sitio TPV1. Este hallazgo es coherente con las pocas evidencias contemporáneas de maíz para el NOA y norte de Chile cuyos contextos no pueden adscribirse como domésticos en el sentido más tradicional, es decir que ninguno está asociado a áreas de actividades específicas relacionadas con el procesamiento o cocción de alimentos. De hecho, granos de maíz reportados para la Quebrada de Tulán (Chile) con una cronología de 2480-2380 años AP fueron interpretados como ofrendas en entierros de neonatos (Núñez et al., 2017). El caso espacialmente más cercano a QDLC del montículo de El Mollar, donde se han recuperado numerosos granos de maíz, habría tenido también una funcionalidad ritual (González y Núñez Regueiro 1960; Carrizo et al., 1999).

Además de maíz, tanto en QDLC como en los otros sitios tempranos mencionados, se ha registrado la presencia de semillas de quínoa. Junto con este taxón, proponemos que el maíz en TPV1 formó parte de un contexto ritual/funerario, que en este caso involucró prácticas crematorias. Los granos de maíz y de quínoa fueron depositados como ofrendas o como acompañamiento de los muertos. Y eventualmente pudieron haber sido parte de un consumo ritual que habría involucrado también a los vivos, compartiendo un mismo alimento, un momento ritual entrelazado con una actividad cotidiana como es la alimentación. En este caso, esto implicaría una preparación especial, agregando al fuego de los muertos un ingrediente que en ese momento -hacia ca. 3500 AP- no era aún de uso generalizado como alimento. Comer junto al fuego de cremación y alimentarlo implica una unión, al menos momentánea, entre los participantes del banquete, ya sean los vivos, los muertos o el fogón en sí. Esto es coherente con lo que Capparelli (2015) denomina como comensalidad ampliada, donde seres de distinto orden y diversos componentes (materiales, paisajísticos) se ven involucrados en el acto comensal. Siguiendo esta línea, dos mil años después los muertos serían enterrados en los patios de las viviendas, es decir en el centro del ámbito doméstico continuando con esta tradición de vincular a la vida y la muerte en una dimensión única (Salazar, 2012; Oliszewski et al., 2018).

Consideramos que la presencia de estos granos hacia 3500 AP en QDLC abre un interesante panorama a explorar en relación a una fase inicial de manejo in situ de estas especies en el seno de sociedades básicamente no-productoras de alimentos. Lo que sí queda claramente demostrado, es que estas especies domesticadas tuvieron inicialmente una mayor vinculación hacia la esfera de lo ritual/funerario que a lo estrictamente alimenticio, sin descartar la posibilidad de que se trate de elementos suntuarios de origen exótico dada la movilidad que aún caracterizaba a los grupos que habitaron QDLC hacia finales del Holoceno medio. Para los inicios de la Era Cristiana, QDLC ya estaba habitada por grupos completamente sedentarios que cultivaban estas plantas a gran escala para el abastecimiento de toda la aldea de Puesto Viejo (Oliszewski et al., 2015).

Por ahora, estas muestras de Z. mays, datadas en ca. 3500 años AP, del sitio TPV1 son los macrorrestos más antiguos para el norte de Argentina y Chile. No obstante, consideramos que el avance de las investigaciones en distintas regiones, devendrá en que el hallazgo aquí presentado sea uno más de muchos otros casos que den cuenta de la larga historia que tiene el maíz en esta parte de los Andes centro-sur.

\section{NOTAS}

${ }^{1}$ El diseño de las estructuras residenciales es el denominado patrón Tafí (sensu Berberián \& Nielsen 1988) en el que las unidades de vivienda están compuestas por pequeñas habitaciones circulares de 2 a $6 \mathrm{~m}$ de diámetro dispuestas alrededor de un núcleo central de 10 a $15 \mathrm{~m}$ de diámetro.

${ }^{2}$ Recientemente fue fechada una de las semillas de quínoa (Chenopodium quinoa) de la capa temprana, la cual proporcionó una datación de ca. 3040 años AP (Oliszewski et al., 2018). 


\section{AGRADECIMIENTOS}

Agradecemos a las siguientes instituciones que subsidiaron nuestras investigaciones: National Geographic Society (Grants 9581-14 y 9930-16), Agencia Nacional de Promoción Científica y Tecnológica (PICT 2013-1700), Consejo Nacional de Investigaciones Científicas y Técnicas (PIP 0141 y PIP 0222) y Secretaría de Ciencia, Arte e Innovación Tecnológica de la Universidad Nacional de Tucumán (PIUNT G521).

A Fernanda Rodríguez y Virginia Mc Rostie por sus valiosos comentarios.

\section{BIBLIOGRAFÍA}

Aguirre, G. \& M. F. Rodríguez. 2015. Discusiones teóricas y metodológicas en torno a la transición entre la recolección y la agricultura incipiente en Antofagasta de la Sierra, Catamarca. Comechingonia Revista de Arqueología 19(2):159-183.

Arreguez, G.; M. Gramajo Bühler \& N. Oliszewski. 2010. Utilización de recursos vegetales alimenticios en sitios arqueológicos de altura. El caso de Cueva de Los Corrales 1 (El Infiernillo, Tafí del Valle, Tucumán, Argentina), en S. Bertolino, R. Cattaneo, A. Izeta \& G. Castellano (eds.), La Arqueometría en Argentina y Latinoamérica, pp. 211-218. Córdoba: Editorial de la FF y H (UNC).

Arreguez, G.; J. G. Martínez, N. Oliszewski \& G. Ponessa. 2014. La problemática de recuperación de macrorrestos arqueobotánicos de tamaño pequeño. El caso de las amarantáceas/quenopodiáceas en sitios arqueológicos bajo reparo del Holoceno Medio y Tardío del Noroeste Argentino, en C. Belmar \& V. Lema (eds.), Avances y desafios metodológicos en arqueobotánica. Miradas consensuadas $y$ diálogos compartidos desde Sudamérica, Monografías arqueológicas, pp. 56-68. Santiago de Chile: Facultad de Patrimonio cultural y Educación. Universidad SEK.

Arriaza, B.; J. P. Ogalde, J. Chacama, V. Standen, L. Huamán \& F. Villanueva. 2015. Estudio de almidones en queros de madera del Norte de Chile relacionados con el consumo de chicha durante el Horizonte Inca. Estudios Atacameños. Arqueología y Antropologías Surandinas 50: 59-84.

Babot, M. del P. 2007. Granos de almidón en contextos arqueológicos: posibilidades y perspectivas a partir de casos del Noroeste argentino, en B. Marconetto, M. del P. Babot \& N. Oliszewski (eds.), Investigaciones arqueobotánicas en Latinoamérica: estudios de casos y propuestas metodológicas, pp. 95-126. Córdoba: Editorial FF y H (UNC).
Babot, M. del P. 2003 Starch grain damage as an indicator of food processing, en D. Hart D. \& L. Wallis (eds.), Phytolith and starch research in the Australian-PacificAsian regions: the state of the art. Terra Australis 19, pp. 69-81. Canberra: Pandamus Books for the Centre for Archaeological Research (ANU).

Babot, M. del P. 2006. El papel de la molienda en la transición hacia la producción agropastoril: Un análisis desde la Puna Meridional argentina. Estudios Atacameños. Arqueología y Antropología Surandinas 32: 75-92.

Babot, M. del P. 2011. Cazadores-recolectores de los Andes Centro-Sur y procesamiento vegetal. Una discusión desde la Puna Meridional Argentina (ca. 7000-3200 años A.P.). Chungara Revista de Antropología Chilena 43(1): 413-432.

Berberián, E. E. \& A. Nielsen. 1988. Sistemas de asentamiento prehispánico en la etapa Formativa del valle de Tafí (Pcia. de Tucumán- Rep. Arg.), en E. E. Berberián (ed.) Sistemas de Asentamiento Prehispánicos en el Valle de Tafi, pp. 2151. Córdoba: Editorial Comechingonia.

Capparelli, A. 2015. La arqueobotánica del sitio Inka "El Shincal de Quimivil" durante la última década: interpretación de prácticas culinarias dentro de un marco de comensalidad ampliada, en R.A. Raffino, L. A. Iácona, R. A. Moralejo, D. Gobbo \& M. G. Cousso (eds.), Una capital Inka al sur del Kollasuyu: El Shincal de Quimivil, pp. 63-84. Buenos Aires: Fundación de Historia Natural Félix de Azara.

Carrizo, J.; S. Cano \& M. Soler Nixdorff. 1999. Recursos vegetales comestibles en el Valle de Tafí durante el Período Formativo: análisis arqueobotánico del sitio Casas ViejasE1 Mollar (STucTav2). Actas del XII Congreso Nacional de Arqueología Argentina, 22-26 de septiembre de 1997, La Plata, Tomo I: 65-73.

Carrizo, J.; N. Oliszewski \& S. Cano. 2002. Hallazgo e interpretación de Zea mays en el sitio arqueológico Loma Pircada (La Rioja, Argentina). Publicación Especial $N^{\circ} 8$ de la Asociación Paleontológica Argentina: 79-83.

Gómez Augier, J.; N. Oliszewski \& M. Caria. 2008. Altitude cultivation: phytolith analysis in archaeological farming structure of Quebrada del Río de Los Corrales site (El Infiernillo, Tucumán, Argentina). International Meeting on Phytolith Research, 4th Southamerican Meeting Phytolith Research: 64. Mar del Plata.

González, R. \& V. Núñez Regueiro. 1960. Informe preliminar sobre la investigación arqueológica en Tafí del valle (Noroeste de Argentina). Actas del XXXIV Congreso Internacional de Americanistas, 18-25 de julio de 1960, Viena, pp. 485-496.

Grobman A.; D. Bonavia, T. D. Dillehay, D. R. Piperno, J. Iriarte \& I. Holst. 2012. Preceramic maize from Paredones and Huaca Prieta, Perú. Proceedings of the National Academy of Sciences of the United States of America 109: 1755-1759. 
Franco Salvi, V. L.; M. L. López \& R. Molar. 2014. Microrrestos vegetales en campos de cultivo del primer milenio de la Era en el valle de Tafí (Prov. de Tucumán, República Argentina). Arqueología Iberoamericana 21: 5-29.

Korstanje, M. A. \& M. del P. Babot. 2007. Microfossils characterization from south Andean economic plants, en M. Madella \& D. Zurro (eds.), Plants, people and places. Recents studies in phytolith analysis, pp. 41-72. Oxford: Oxbow Books.

Korstanje, M. A. \& P. Cuenya. 2010. Ancient agriculture and domestic activities in north western Argentina: a contextual approach studying silica phytoliths and other microfossils in soils. Environmental Archaeology. The Journal of Human Paleoecology 15: 43-63.

Lantos, I.; M. Giovanetti \& N. Ratto. 2014. Alcances y limitaciones para la identificación arqueológica de gránulos de almidón de razas nativas de Zea mays (Poaceae) del noroeste argentino. Darwiniana Nueva Serie 2(1): 74-95.

Lema, V. 2014. Boceto para un esquema: domesticación y agricultura temprana en el Noroeste argentino. Revista Española de Antropología Americana 44(2): 465-494.

Martínez, J. G.; E. Mauri, C. Mercuri, M. Caria \& N. Oliszewski. 2013. Mid-Holocene human occupations in Tucumán (Northwest of Argentina). Quaternary International 307: 86-95.

Martínez, J. G.; M. Caria, A. Grau, A. Izeta, N. Olizsewski, E. Pintar \& P. Tchilinguirian. 2016. Final Report National Geographic Society's Committee for Research and Exploration Grant Number: 9581-14. Project "The earliest hunter-gatherers groups in the valleys of NW Argentina: a history of more than 7000 years". Manuscrito en posesión de los autores.

Martínez, J. G.; N. Oliszewski, C. Aranda, L. Luna, E. Naharro \& M. Pérez. 2017. Prácticas en torno a la muerte en la Quebrada de Los Corrales, El Infiernillo, Tucumán (3800-1500 años AP). Libro de Resúmenes XIII Jornadas Nacionales de Antropología Biológica, 13-16 de noviembre de 2017, Necochea, Buenos Aires, pp. 45.

McRostie, V. 2014. Arboricultura y silvopastoralismo en el Período Formativo (1.400 a.C.-500 d.C.) de la cuenca del Salar de Atacama. Chungará Revista de Antropología Chilena 46(4): 543-557.

Núñez, L.; V. McRostie \& I. Cartajena. 2009. Consideraciones sobre la recolección vegetal y la horticultura durante el formativo temprano en el sureste de la cuenca de Atacama. Darwiniana 47: 56-75.

Núñez L.; I. Cartajena, C. Carrasco, P. López, P. de Souza, F. Rivera \& B. Santander. 2017. Presencia de un centro ceremonial formativo en la circumpuna de Atacama. Chungará Revista de Antropología Chilena 49(1): 3-33.
Oliszewski, N. 2005. Archaeobotany of archaeological sites from Northwest Argentina (1750-1450 B.P.): ceremonial use or rubbish dumps?. Vegetation History and Archaeobotany 14(4): 465-471.

Oliszewski, N. 2009. El recurso maíz en sitios arqueológicos del noroeste argentino: el caso de la Quebrada de Los Corrales, El Infiernillo, Tucumán. Treballs d'Etnoarqueologia 7: 83-96.

Oliszewski, N. 2012. La variabilidad racial del maíz y los cambios sociales durante el $1^{\circ}$ milenio d. C. en el noroeste argentino, en M. del P. Babot, F. Pazzarelli \& M. Marschoff (eds), Las manos en la masa. Arqueologías, Antropologías y otras Historias de la alimentación en Suramérica, pp. 271-297. Córdoba: Imprenta Corintios 13.

Oliszewski, N. 2017. Las aldeas "Patrón Tafí" del sur de Cumbres Calchaquíes y norte del Sistema del Aconquija. Comechingonia Revista de Arqueología 21(1): 205-232.

Oliszewski, N. \& G. Arreguez. 2015. Los recursos vegetales alimenticios de la Quebrada de Los Corrales en El Infiernillo, Tucumán, durante el $1^{\circ}$ milenio d.C. Comechingonia Revista de Arqueología 19(2): 111-140.

Oliszewski, N. \& J. G. Martínez. 2017. Primeros resultados sobre paleodietas humanas en la Quebrada de Los Corrales, El Infiernillo, Tucumán (ca. 3300-1550 años AP). Libro de Resúmenes II Taller de Arqueología e Isótopos Estables en el Sur de Sudamérica, 1-3 de noviembre de 2017, San Rafael, Mendoza, pp. 57.

Oliszewski, N.; J. G. Martínez \& M. Caria. 2008. Ocupaciones prehispánicas de altura: el caso de Cueva de los Corrales 1 (El Infiernillo, Tafí del Valle, Tucumán). Relaciones de la Sociedad Argentina de Antropología XXXIII: 209-221.

Oliszewski, N.; J. G. Martínez, G. Arreguez, M. Gramajo Bühler \& E. Naharro. 2018. "La transición" vista desde los valles intermontanos del noroeste argentino: nuevos datos de la Quebrada de Los Corrales (El Infiernillo, Tucumán, Argentina). Chungará Revista de Antropología Chilena 50(1): 71-86.

Oliszewski, N.; J. G. Martínez, E. Di Lullo, M. Gramajo Bühler, G. Arreguez, H. Cruz, E. Mauri, C. Mercuri, A. Muntaner \& G. Srur. 2015. Contribuciones al estudio de sociedades aldeanas en el noroeste argentino: el caso de la quebrada de Los Corrales (E1 Infiernillo, Tucumán), en M. A. Korstanje, M. Lazzari, M. Basile, F. Bugliani, V. Lema, L. Pereyra Domingorena \& M. Quesada (eds.), Crónicas materiales precolombinas: arqueología de los primeros poblados del Noroeste Argentino, pp. 51-79. Buenos Aires, Sociedad Argentina de Antropología.

Parodi, L. 1959. Enciclopedia Argentina de Agricultura y Jardinería 1. Buenos Aires, ACME. 
N. OLISZEWSKI ET AL. Zea mays (Poaceae) en contextos prehispánicos tempranos (Tucumán, Argentina)

Piperno, D. 2006. Phytoliths. A comprehensive guide for archaeologists and paleoecologists. Lanham, Altamira Press.

Pearsall, D. 1989. Paleoethnobotany. A Handbook of procedures. California, Academic Press.

Ratto, N.; V. Lema \& M. L. López. 2014. Entierros y ofrendas: prácticas mortuorias, agrícolas y culinarias en los siglos XIII y XIV en Tinogasta (Catamarca, Argentina). Darwiniana Nueva Serie 2(1):125-143.

Rodríguez, M. F. \& C. A. Aschero. 2007. Archaeological evidence of Zea mays L. (Poaceae) in the Southern
Argentinean Puna (Antofagasta de la Sierra, Catamarca). Journal of Ethnobiology 27(2): 256-271.

Salazar, J. 2012. Household mortuary practices in a South Andean village (first millennium AD). Andean Past 10: 295-302.

Torregrosa M.; J. Cámara Hernández, L. Solari, L. J. Safont \& O. Cavalieri. 1980. Clasificación preliminar de formas raciales de maíz y su distribución geográfica en la República Argentina, Actas del II Congreso Nacional de Maiz, 28 de octubre al 1 de noviembre de 1980, Pergamino (Buenos Aires), pp. 5-17. 